\title{
Transverse oscillations of non-planar coronal loops
}

\author{
M. S. Ruderman and A. Scott
}

\begin{abstract}
School of Mathematics and Statistics, University of Sheffield, Hicks Building, Hounsfield Road, Sheffield S3 7RH, UK e-mail: m.s.ruderman@sheffield.ac.uk
\end{abstract}

Received 19 November 2010 / Accepted 22 February 2011

\section{ABSTRACT}

\begin{abstract}
We suggested a simple model of a non-planar coronal loop (i.e. a loop with torsion). In this model the loop axis is a part of a helical line. Using curvilinear coordinates where the loop axis is a coordinate line and the loop boundary is a coordinate surface we derived the governing equation for the loop kink oscillations. When doing so we have used asymptotic method with the ratio of the loop cross-section radius to the loop curvature radius as a small parameter. The governing equation is exactly the same as one obtained for kink oscillations of a thin straight magnetic tube with the density varying along the tube. This implies that neither the loop curvature nor the loop torsion can directly affect the eigenfrequencies of the loop kink oscillations. They can affect these eigenfrequencies only indirectly through modifying the dependence of the density on the distance along the loop. The main effect of the loop torsion is on the polarization of the loop oscillations. We found that, when we are moving along the loop, the polarization direction is rotating together with the principal normal to the loop axis due to the loop torsion. The application of the obtained results to the interpretation of observations of the loop kink oscillations with a node is discussed.
\end{abstract}

Key words. magnetohydrodynamics (MHD) - waves - Sun: oscillations - Sun: corona

\section{Introduction}

After the first observation of transverse coronal loop oscillations by TRACE in 1998 this phenomenon remains in the focus of attention of solar physicists. In the first theoretical studies of this phenomenon a very simple model of a coronal loop was used (see Ryutov \& Ryutova 1976; Edwin \& Roberts 1983). In this model a coronal loop is considered as a straight homogeneous magnetic cylinder. Later, more sophisticated models of coronal loops were developed. These models take into account such properties of the loops as the curvature, the density variation along and across the loop, and the loop non-circular crosssection. For a recent review of the theory of transverse oscillations of coronal loops see, e.g., Ruderman \& Erdélyi (2009). However, in all these theories the loop was assumed to be confined to a plane.

Recently Aschwanden et al. (2008) have used data from the STEREO spacecraft to triangulate the positions of several coronal loops and reconstruct their 3D geometry. As a part of their analysis they considered whether the loops were planar and found that seven loops were not confined to a plane. Hence, these loops were not only curved, but they also had non-zero torsion. This discovery puts on the agenda studying transverse oscillations of non-planar coronal loops.

In this paper we present the first theoretical study of kink oscillations of non-planar loops. The paper is organized as follows. In the next section we describe the equilibrium for the nonplanar coronal loop used in our study. In Sect. 3 we introduce the curvilinear coordinates used to derive the equation governing the loop kink oscillations. In Sect. 4 we derive the linear ideal MHD equations and boundary conditions in the curvilinear coordinates. In Sect. 5 we give the derivation of the governing equation. In Sect. 6 we study the variation of polarization of the kink oscillation along the loop. In Sect. 7 we discuss possible applications of the developed theory to coronal seismology.

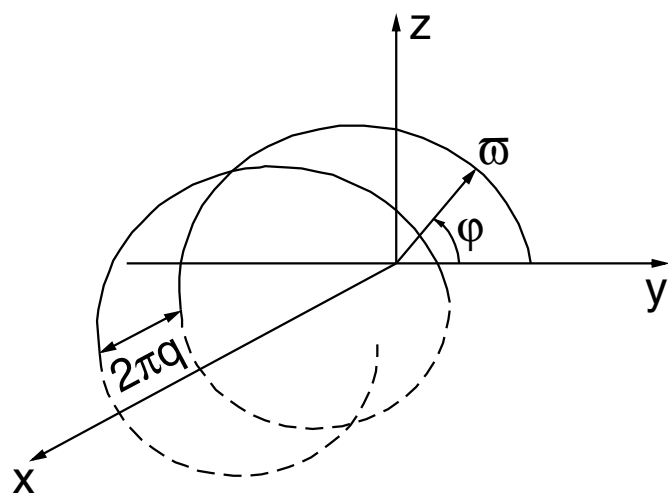

Fig. 1. The auxiliary Cartesian and cylindrical coordinates. A typical magnetic field line is shown. Parts of this line that are above the $x y$-plane are shown by solid lines, and those below the $x y$-plane by dashed lines.

Section 8 contains the summary of the obtained results and our conclusions.

\section{Equilibrium}

Let us introduce Cartesian coordinates $x, y, z$ with the $z$-axis in the vertical direction, and cylindrical coordinates $\varpi, \varphi, x$ (see Fig. 1). The Cartesian and cylindrical coordinates are related by

$x=x, \quad y=\varpi \cos \varphi, \quad z=\varpi \sin \varphi$.

Consider the magnetic field $\boldsymbol{B}$ with the components given by

$B_{x}=\frac{q^{2} B_{0}}{q^{2}+\varpi^{2}}, \quad B_{\varpi}=0, \quad B_{\varphi}=\frac{q \varpi B_{0}}{q^{2}+\varpi^{2}}$, 
where $q$ is a constant. It is straightforward to obtain that

$\nabla \times \boldsymbol{B}=\frac{2 q \boldsymbol{B}}{q^{2}+\varpi^{2}}$.

It follows from this result that the magnetic field $\boldsymbol{B}$ is force-free and can be used as an equilibrium magnetic field if we adopt the cold plasma approximation. The parametric equations of the magnetic field lines are given by

$$
x=q \varphi+x_{0}, \quad y=\varpi_{0} \cos \varphi, \quad z=\varpi_{0} \sin \varphi,
$$

where $x_{0}$ and $\varpi_{0}$ are constants. All the magnetic field lines are spirals with the same pitch equal to $2 \pi q$. This means that each magnetic field line is invariant under the helical space transformation defined by

$\varphi \mapsto \varphi+\tilde{\varphi}, \quad x \mapsto x+q \tilde{\varphi}$,

where $\tilde{\varphi}$ is an arbitrary constant. The magnetic field magnitude is $q B_{0}\left(q^{2}+\varpi^{2}\right)^{-1 / 2}$. Since $\varpi=$ const. on a magnetic field line, the magnetic field magnitude is also constant.

The magnetic field lines are frozen in the dense photospheric plasma at $z=z_{0}$. One of the magnetic field lines corresponding to $x_{0}=0$ and $\varpi_{0}=R>\left|z_{0}\right|$ in Eq. (4) is the magnetic loop axis. Its equation is

$x=q \varphi, \quad y=R \cos \varphi, \quad z=R \sin \varphi, \quad \varphi \in\left[\varphi_{0}, \pi-\varphi_{0}\right]$,

where $\varphi_{0}=\arcsin \left(z_{0} / R\right)$. The loop axis crosses the plane $z=z_{0}$ at points with the coordinates

$\left(q \varphi_{0}, \sqrt{R^{2}-z_{0}^{2}}, z_{0}\right), \quad\left(q \pi-q \varphi_{0},-\sqrt{R^{2}-z_{0}^{2}}, z_{0}\right)$.

The curvature and torsion of the loop axis are equal to $R\left(q^{2}+\right.$ $\left.R^{2}\right)^{-1}$ and $q\left(q^{2}+R^{2}\right)^{-1}$ respectively. We should point out that one needs to make a clear distinction between the loop torsion and twist. The loop torsion is related to the shape of the loop axis. It measures the loop non-planarity. The twist is related to the behaviour of the magnetic field lines in the vicinity of the loop axis. A straight loop is twisted if the magnetic field lines are helical in the vicinity of its axis. How to distinguish between twisted and non-twisted magnetic loops when they are not straight? We know that, in the case of a straight loop, the magnetic twist creates the electrical current along the loop. We can extend this relation between the magnetic twist and electrical current to curved loops and give the following definition: a magnetic loop is twisted if there is the electrical current parallel to the loop axis and untwisted otherwise. It follows from Eq. (3) that there is the electrical current parallel to the loop axis in our model. Hence, in the equilibrium that we consider here, the magnetic loop is twisted.

The loop axis (or its extension if $z_{0}>0$ ) crosses the $x y$ plane at the point $(0, R)$. Let us take the plane $\Pi_{0}$ orthogonal to the loop axis (or its extension) at this point and consider the circle $C_{0}$ of radius $a$ centered at $(0, R), a \ll R$. The magnetic field lines crossing the plane $\Pi_{0}$ at points on the circle $C_{0}$ form the tube boundary. The plasma density is equal to $\rho_{i}$ inside the loop and $\rho_{e}$ outside. Both $\rho_{i}$ and $\rho_{e}$ can vary along the loop, but they do not vary in the directions perpendicular to the loop axis. Note that we obtain a particular case of a loop with the shape of a half-torus if we take $q=0$ and $\varphi_{0}=0$. The kink oscillations of such a loop have been studied by Van Doorsselaere et al. (2004) analytically and Terradas et al. (2006) numerically (see also the review by Van Doorsselaere et al. 2009).

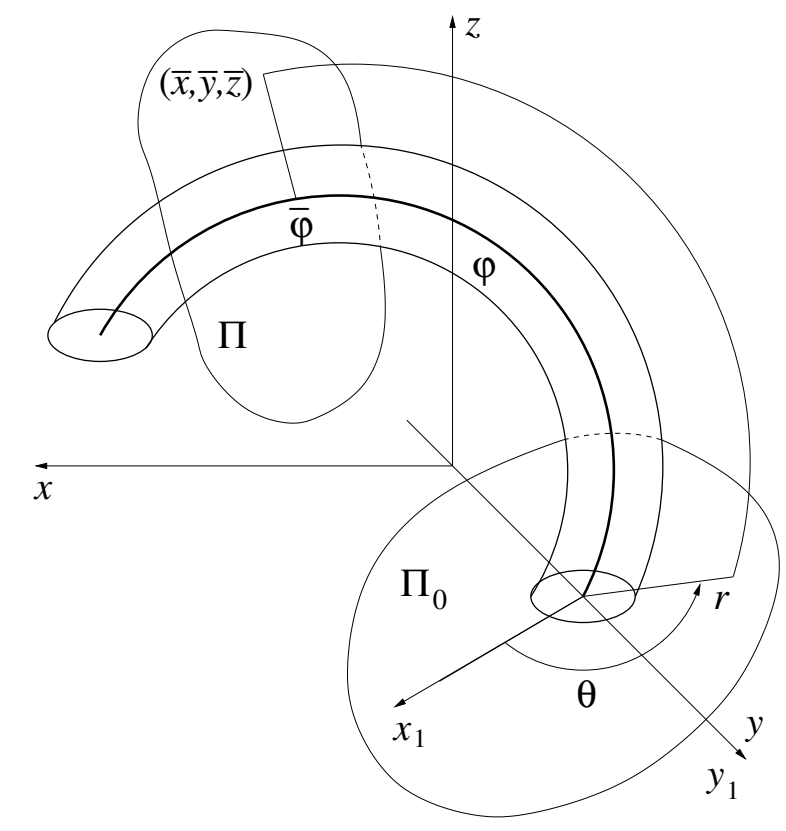

Fig. 2. The sketch of the equilibrium state. The ends of the magnetic tube are assumed to be frozen in a dense photospheric plasma. The axis of the magnetic loop is shown by the thick line. Introducing curvilinear coordinates is illustrated.

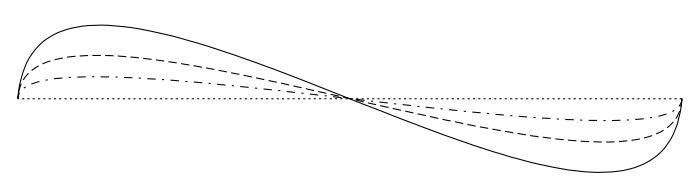

Fig. 3. The projection of a loop on the horizontal $x y$-plane. The solid, dashed and dashed-dotted lines correspond to $q / R=0.2,0.1$ and 0.05 respectively.

Let us make the helical space transformation defined by Eq. (5). Recall that any magnetic field line is invariant under this transformation. In particular, this implies that the magnetic field line containing the tube axis is mapped in itself. Since the helical transformation is an orthogonal transformation, it maps the plane $\Pi_{0}$ into a plane $\Pi$ that is orthogonal to the loop axis at the point of their intersection. The circle $C_{0}$ is mapped in a circle $C$ of radius $a$ in the plane $\Pi$ centered at the point of intersection of $\Pi$ with the loop axis. Since any magnetic field line is mapped into itself, any point on $C$ is at the same time on the tube boundary. Hence, $C$ is the intersection of $\Pi$ with the loop boundary, which implies that the loop cross-section is everywhere a circle of radius $a$ centered at the loop axis. The magnetic loop is schematically shown in Fig. 2. In Fig. 3 the projections of the loops on the horizontal plane are shown for different values of $q / R$. We can see that these projections resemble the letter $\mathrm{S}$, which is why the non-planar loops are often called the S-shape loops.

\section{Curvilinear coordinates}

We introduce the small parameter $\varepsilon=a / R$. In what follows we only consider perturbations that decay far from the loop with the characteristic scale of decay equal to $a$. Hence we study the plasma motion only inside the loop and in the loop vicinity outside the loop. As a result we need the curvilinear coordinates that only cover the spatial domain elongated in the direction of 
the loop axis and with the size of a few $a$ in the directions orthogonal to the loop axis.

To obtain the curvilinear coordinate system we first introduce polar coordinates in the $\Pi_{0}$ plane. It follows from the parametric equation of the loop axis Eq. (6) that the vector

$\boldsymbol{l}=(q,-R \sin \varphi, R \cos \varphi)$

is tangent to the loop axis or its extension. Then the equation of the plane $\Pi_{0}$ is

$q x+R z=0$.

Introduce new Cartesian coordinates $x_{1}, y_{1}, z_{1}$ related to the old ones by

$x_{1}=\frac{R x-q z}{\sqrt{R^{2}+q^{2}}}, \quad y_{1}=y, \quad z_{1}=\frac{q x+R z}{\sqrt{R^{2}+q^{2}}}$.

In the new coordinates the equation of plane $\Pi_{0}$ is $z_{1}=0$, so that $x_{1}$ and $y_{1}$ are Cartesian coordinates in $\Pi_{0}$. Now we introduce the polar coordinates $r$ and $\theta$ in $\Pi_{0}$ related to $x_{1}$ and $y_{1}$ by

$x_{1}=r \cos \theta, \quad y_{1}=R+r \sin \theta$.

In these polar coordinates the equation of circle $C_{0}$ of radius $a \ll$ $R$ in the plane $\Pi_{0}$ centered at $x_{1}=0, y_{1}=R$ is $r=a$. Inverting the relations Eq. (10) we obtain that, for any point $(x, y, z) \in \Pi_{0}$,

$x=\frac{R r \cos \theta}{\sqrt{R^{2}+q^{2}}}, \quad y=R+r \sin \theta, \quad z=-\frac{q r \cos \theta}{\sqrt{R^{2}+q^{2}}}$.

To obtain the curvilinear coordinates of arbitrary point $(\bar{x}, \bar{y}, \bar{z})$ we do the following. First we take the magnetic field line $\mathcal{L}$ that passes through this point. It intersects the plane $\Pi_{0}$ at the point with the polar coordinates $r, \theta$ (see Fig. 2). These are the first and second curvilinear coordinates of the point $(\bar{x}, \bar{y}, \bar{z})$. To obtain the third curvilinear coordinate we take the plane $\Pi$ orthogonal to the loop axis and containing $(\bar{x}, \bar{y}, \bar{z})$. It crosses the loop axis at the point corresponding to the value $\bar{\varphi}$ of the parameter $\varphi$. Then the curvilinear coordinates of $(\bar{x}, \bar{y}, \bar{z})$ are $(r, \theta, \bar{\varphi})$.

Now we obtain the expressions of $\bar{x}, \bar{y}$ and $\bar{z}$ in terms of $r$, $\theta$ and $\bar{\varphi}$. Let $\mathcal{L}$ cross $\Pi_{0}$ at the point $\left(x_{c}, y_{c}, z_{c}\right)$. The parametric equation of $\mathcal{L}$ is given by Eq. (4) where we will write $\varphi^{\prime}$ instead of $\varphi$ so as not to mix the parameter on $\mathcal{L}$ with the third coordinate of the curvilinear coordinate system. It follows from Eq. (4) that $\bar{y}^{2}+\bar{z}^{2}=\varpi_{0}^{2}=$ const. Then, on $\mathcal{L}$,

$$
\begin{aligned}
\bar{y}^{2}+\bar{z}^{2} & =\varpi_{0}^{2}=y_{c}^{2}+z_{c}^{2}=(R+r \sin \theta)^{2}+\frac{q^{2} r^{2} \cos ^{2} \theta}{R^{2}+q^{2}} \\
& =R^{2}+2 R r \sin \theta+r^{2} \frac{q^{2}+R^{2} \sin ^{2} \theta}{R^{2}+q^{2}} .
\end{aligned}
$$

$\mathcal{L}$ intersects the plane $\Pi_{0}$ when $\varphi^{\prime}=\varphi_{c}^{\prime}$. Then, in accordance with Eqs. (4) and (13),

$x_{c}=q \varphi_{c}^{\prime}+x_{0}, \quad y_{c}=\varpi_{0} \cos \varphi_{0}^{\prime}, \quad z_{c}=\varpi_{0} \sin \varphi_{0}^{\prime}$.

On the other hand, it follows from Eq. (12) that

$x_{c}=\frac{R r \cos \theta}{\sqrt{R^{2}+q^{2}}}, \quad y_{c}=R+r \sin \theta, \quad z_{c}=-\frac{q r \cos \theta}{\sqrt{R^{2}+q^{2}}}$.

Using Eqs. (14) and (15) we obtain

$$
\begin{gathered}
x_{0}=\frac{R r \cos \theta}{\sqrt{R^{2}+q^{2}}}-q \varphi_{c}^{\prime}, \quad \cos \varphi_{c}^{\prime}=\frac{R+r \sin \theta}{\varpi_{0}}, \\
\sin \varphi_{c}^{\prime}=-\frac{q r \cos \theta}{\varpi_{0} \sqrt{R^{2}+q^{2}}} .
\end{gathered}
$$

The tangent vector to the loop axis at the point of its intersection with $\Pi$ is given by Eq. (8) with $\bar{\varphi}$ substituted for $\varphi$. Then the equation of this plane is

$q(x-\bar{x})-R \sin \bar{\varphi}(y-\bar{y})+R \cos \bar{\varphi}(z-\bar{z})=0$.

The coordinates of the point of intersection are given by Eq. (6) with $\bar{\varphi}$ substituted for $\varphi$. They satisfy Eq. (17), so that

$q \bar{x}=q^{2} \bar{\varphi}+R(\bar{y} \sin \bar{\varphi}-\bar{z} \cos \bar{\varphi})$.

The point $(\bar{x}, \bar{y}, \bar{z})$ corresponds to $\varphi^{\prime}=\bar{\varphi}^{\prime}$. Then it follows from Eqs. (4) and (16) that

$\bar{x}=q\left(\bar{\varphi}^{\prime}-\varphi_{c}^{\prime}\right)+\frac{R r \cos \theta}{\sqrt{R^{2}+q^{2}}}, \bar{y}=\varpi_{0} \cos \bar{\varphi}^{\prime}, \bar{z}=\varpi_{0} \sin \bar{\varphi}^{\prime}$.

Substituting these expressions in Eq. (18) we obtain the equation determining $\bar{\varphi}^{\prime}$,

$q^{2}\left(\bar{\varphi}^{\prime}-\bar{\varphi}-\varphi_{c}^{\prime}\right)+\varpi_{0} R \sin \left(\bar{\varphi}^{\prime}-\bar{\varphi}\right)=-\frac{q R r \cos \theta}{\sqrt{R^{2}+q^{2}}}$.

This equation cannot be solved analytically. However it does not cause any problem because we do not need this in what follows. As we have already mentioned, we use the curvilinear coordinates only in the vicinity of the loop, at distances not exceeding a few $a$. Hence, it suffices to obtain only approximate expressions of Cartesian coordinates in terms of $r, \theta$ and $\varphi$ that are linear with respect to $r$. To do this first we note that, in the zero order approximation, i.e. when $r=0$, it follows from Eqs. (13) and (16) that $\varpi_{0}=R$ and $\varphi_{c}^{\prime}=0$. Then the solution to Eq. (20) is $\bar{\varphi}^{\prime}=\bar{\varphi}$. In fact, this result is obvious because, when $r=0, \mathcal{L}$ coincides with the loop axis and $(\bar{x}, \bar{y}, \bar{z})$ coincides with the point of intersection of $\Pi$ with the loop axis.

In the next order approximation we obtain from Eqs. (13) and (16)

$\varpi_{0} \approx R+r \sin \theta, \quad \varphi_{c}^{\prime} \approx-\frac{q r \cos \theta}{R \sqrt{R^{2}+q^{2}}}$.

Then we look for the approximate solution to Eq. (20) in the form $\bar{\varphi}^{\prime}=\bar{\varphi}+c r$, where $c$ is a constant to be determined. Substituting this expression in Eq. (20) and using Eq. (21) we easily find $c$ to obtain

$\bar{\varphi}^{\prime} \approx \bar{\varphi}-\frac{q r \cos \theta}{R \sqrt{R^{2}+q^{2}}}$.

Substituting Eqs. (21) and (22) in Eq. (19) and dropping the bar we finally arrive at

$$
\begin{aligned}
& x=q \varphi+\frac{R r \cos \theta}{\sqrt{R^{2}+q^{2}}}+O\left(\varepsilon^{2}\right), \\
& y=R \cos \varphi+r \sin \theta \cos \varphi+\frac{q r \cos \theta \sin \varphi}{\sqrt{R^{2}+q^{2}}}+O\left(\varepsilon^{2}\right), \\
& z=R \sin \varphi+r \sin \theta \sin \varphi-\frac{q r \cos \theta \cos \varphi}{\sqrt{R^{2}+q^{2}}}+O\left(\varepsilon^{2}\right),
\end{aligned}
$$

where $\varepsilon=a / R$ and $O\left(\varepsilon^{2}\right)$ indicates higher terms with respect to $r$ starting from quadratic. The relations Eq. (23) will be used in the next section to write down the linearized equations of the ideal MHD in the curvilinear coordinates. 


\section{Equations and boundary conditions}

The aim of this section is to derive the governing equations and boundary conditions written in the curvilinear coordinates. We start this derivation from introducing the stretching variable $\sigma=\varepsilon^{-1} r / R$. It is convenient to introduce this variable because, in what follows, we consider only the vicinity of the magnetic tube where $r / R=O(\varepsilon)$. Hence, from now on, the curvilinear coordinates are $\sigma, \theta, \varphi$. To be able to use the Einstein summation rule for repeating indices we also introduce another notation for the curvilinear coordinates,

$u^{1}=\sigma, \quad u^{2}=\theta, \quad u^{3}=\varphi$.

Now we need to calculate the components of the metric tensor. Let us introduce vector $\boldsymbol{X}=(x, y, z)$, and calculate the derivatives of $\boldsymbol{X}$ with respect to $u^{i}$. Using Eq. (23) we obtain

$$
\begin{array}{r}
\frac{\partial X}{\partial \sigma}=\varepsilon R\left(\frac{R \cos \theta}{\sqrt{R^{2}+q^{2}}}, \sin \theta \cos \varphi+\frac{q \cos \theta \sin \varphi}{\sqrt{R^{2}+q^{2}}}\right. \\
\left.\sin \theta \sin \varphi-\frac{q \cos \theta \cos \varphi}{\sqrt{R^{2}+q^{2}}}\right)+O\left(\varepsilon^{2}\right)
\end{array}
$$

$$
\begin{array}{r}
\frac{\partial \boldsymbol{X}}{\partial \theta}=\varepsilon \sigma R\left(-\frac{R \sin \theta}{\sqrt{R^{2}+q^{2}}}, \cos \theta \cos \varphi-\frac{q \sin \theta \sin \varphi}{\sqrt{R^{2}+q^{2}}}\right. \\
\left.\cos \theta \sin \varphi+\frac{q \sin \theta \cos \varphi}{\sqrt{R^{2}+q^{2}}}\right)+O\left(\varepsilon^{2}\right)
\end{array}
$$

$$
\begin{aligned}
& \frac{\partial X}{\partial \varphi}=\left(q,-R \sin \varphi-\varepsilon \sigma R \sin \theta \sin \varphi+\frac{\varepsilon \sigma q R \cos \theta \cos \varphi}{\sqrt{R^{2}+q^{2}}}\right. \\
& \left.R \cos \varphi+\varepsilon \sigma R \sin \theta \cos \varphi+\frac{\varepsilon \sigma q R \cos \theta \sin \varphi}{\sqrt{R^{2}+q^{2}}}\right)+O\left(\varepsilon^{2}\right)
\end{aligned}
$$

Using Eqs. (25)-(27) and recalling that

$g_{i j}=\frac{\partial X}{\partial u^{i}} \cdot \frac{\partial X}{\partial u^{j}}$

we obtain the following expressions for the covariant components of the metric tensor,

$$
\begin{array}{ll}
g_{11}=\varepsilon^{2} R^{2}+O\left(\varepsilon^{3}\right), & g_{12}=O\left(\varepsilon^{3}\right), \\
g_{13}=O\left(\varepsilon^{2}\right), & g_{22}=\varepsilon^{2} \sigma^{2} R^{2}+O\left(\varepsilon^{3}\right), \\
g_{23}=O\left(\varepsilon^{2}\right), & g_{33}=R^{2}+q^{2}+O(\varepsilon) .
\end{array}
$$

We see that the curvilinear coordinate system is non-orthogonal. The covariant components of the metric tensor are the elements of a $3 \times 3$ matrix. The determinant of this matrix is given by

$g=\varepsilon^{4} \sigma^{2} R^{4}\left(R^{2}+q^{2}\right)+O\left(\varepsilon^{5}\right)$.

In what follows we use the co- and contravariant components of vector $\boldsymbol{\xi},\left(\xi_{1}, \xi_{2}, \xi_{3}\right)$ and $\left(\xi^{1}, \xi^{2}, \xi^{3}\right)$, and the same for vectors $\boldsymbol{b}$ and $\boldsymbol{B}$. It is obvious from the description of the curvilinear coordinate system that any magnetic field line is defined by equations $\sigma=$ const. and $\theta=$ const., so that any magnetic field line is a $\varphi$-coordinate line. Since $\boldsymbol{B}$ is tangent to a magnetic field line, it follows that its contravariant components are

$B^{1}=0, \quad B^{2}=0, \quad B^{3}=\frac{B}{\sqrt{g_{33}}}$, where $B$ is the magnetic field magnitude given by

$B=\frac{q B_{0}}{\sqrt{q^{2}+\varpi^{2}}}=\frac{q B_{0}}{\sqrt{q^{2}+R^{2}}}+O(\varepsilon)$.

Using Eqs. (28) and (31) we obtain

$B^{3}=\frac{q B_{0}}{q^{2}+R^{2}}+O(\varepsilon)$

Now it follows from the identity $|\boldsymbol{B}|^{2}=B_{i} B^{i}=B_{3} B^{3}$ that

$B_{3}=q B_{0}+O(\varepsilon)$.

In our analysis we will use the expression for the vector product of two vectors, and for a curl of an arbitrary vector (e.g. Korn \& Korn 1961; Riley et al. 2002)

$(\boldsymbol{v} \times \boldsymbol{w})_{i}=\sqrt{g} \epsilon_{i j k} v^{j} w^{k}, \quad(\nabla \times \boldsymbol{v})^{i}=\frac{1}{\sqrt{g}} \epsilon^{i j k} \frac{\partial v_{k}}{\partial u^{j}}$.

Here $\epsilon_{i j k}=\epsilon^{i j k}$ are the Levi-Civita symbols. Recall that $\epsilon_{i j k}= \pm 1$ if all the indices are different, and $\epsilon^{i j k}=0$ if at least two indices are equal.

Let us introduce the physical components of the displacement vector, $\boldsymbol{\xi}=\left(\xi_{r}, \xi_{\theta}, \xi_{\varphi}\right)$. These components are related to the contravariant components by

$\xi_{r}=\sqrt{g_{11}} \xi^{1}, \quad \xi_{\theta}=\sqrt{g_{22}} \xi^{2}, \quad \xi_{\varphi}=\sqrt{g_{33}} \xi^{3}$.

In what follows we consider $\xi_{r}$ and $\xi_{\theta}$ as quantities of the order of unity. Then it follows from Eq. (28) that $\xi^{1}=O\left(\varepsilon^{-1}\right)$ and $\xi^{2}=O\left(\varepsilon^{-1}\right)$. These estimates inspire us to introduce the scaled contravariant components of the displacement,

$\bar{\xi}^{1}=\varepsilon \xi^{1}, \quad \bar{\xi}^{2}=\varepsilon \xi^{2}$.

For the covariant components we have with the aid of Eq. (28) that

$\xi_{1}=g_{1 j} \xi^{j}=O(\varepsilon), \quad \xi_{2}=g_{2 j} \xi^{j}=O(\varepsilon)$.

In accordance with these estimates we introduce the scaled covariant components of the displacement,

$\bar{\xi}_{1}=\varepsilon^{-1} \xi_{1}, \quad \bar{\xi}_{2}=\varepsilon^{-1} \xi_{2}$.

To describe the plasma motion we use the ideal linear MHD equations in the cold plasma approximation,

$\rho \frac{\partial^{2} \boldsymbol{\xi}}{\partial t^{2}}=\frac{1}{\mu_{0}}(\nabla \times \boldsymbol{b}) \times \boldsymbol{B}+\frac{1}{\mu_{0}}(\nabla \times \boldsymbol{B}) \times \boldsymbol{b}$,

$\boldsymbol{b}=\nabla \times(\boldsymbol{\xi} \times \boldsymbol{B})$.

It follows from Eq. (30) and the first equation in Eq. (34) that $(\boldsymbol{v} \times \boldsymbol{B})_{3}=0$ for any vector $\boldsymbol{v}$. Then, taking into account Eq. (3), we immediately conclude that the third covariant component of the right-hand side of Eq. (39) is zero. This implies that $\xi_{3}=0$. Now, using the identity $\xi_{3}=g_{31} \xi^{1}+g_{32} \xi^{2}+g_{33} \xi^{3}$ and Eqs. (28) and (36) we obtain $\xi^{3}=O(\varepsilon)$.

Using Eqs. (30) and (34), and the identity

$\epsilon^{i j k} \epsilon_{k l m}=\delta_{l}^{i} \delta_{m}^{j}-\delta_{m}^{i} \delta_{l}^{i}$

we obtain from Eqs. (40)

$b^{i}=\frac{1}{\sqrt{g}} \frac{\partial}{\partial u^{3}}\left(B \xi^{i} \sqrt{\frac{g}{g_{33}}}\right)-\frac{\delta_{3}^{i}}{\sqrt{g}} \frac{\partial}{\partial u^{j}}\left(B \xi^{j} \sqrt{\frac{g}{g_{33}}}\right)$. 
M. S. Ruderman and A. Scott: Transverse oscillations of non-planar loops

Let us introduce the perturbation of the magnetic pressure,

$P=\frac{1}{\mu_{0}} \boldsymbol{B} \cdot \boldsymbol{b}=\frac{1}{\mu_{0}} B^{i} b_{i}=\frac{B b_{3}}{\mu_{0} \sqrt{g_{33}}} \cdot$

Using Eq. (42) and the relation $b_{i}=g_{i j} b^{j}$ we obtain

$P=\frac{B}{\mu_{0} \sqrt{g}}\left(\frac{g_{3 i}}{\sqrt{g_{33}}} \frac{\partial}{\partial u^{3}}-\sqrt{g_{33}} \frac{\partial}{\partial u^{i}}\right)\left(B \xi^{i} \sqrt{\frac{g}{g_{33}}}\right)$.

Using Eqs. (28) and (36), and the estimate $\xi^{3}=O(\varepsilon)$ we reduce Eq. (44) to

$$
\begin{aligned}
P=-\frac{\varepsilon^{-1} B}{\mu_{0}} \sqrt{\frac{g_{33}}{g}}[ & \frac{\partial}{\partial u^{1}}\left(B \bar{\xi}^{1} \sqrt{\frac{g}{g_{33}}}\right) \\
& \left.+\frac{\partial}{\partial u^{2}}\left(B \bar{\xi}^{2} \sqrt{\frac{g}{g_{33}}}\right)\right]+O(\varepsilon) .
\end{aligned}
$$

Using Eqs. (34) and (41) we obtain

$[(\nabla \times \boldsymbol{b}) \times \boldsymbol{B}]_{i}=B^{j} \frac{\partial b_{i}}{\partial u^{j}}-B^{j} \frac{\partial b_{j}}{\partial u^{i}}$.

With the aid of Eqs. (30) and (43) we reduce this expression to

$$
\begin{aligned}
{[(\nabla \times \boldsymbol{b}) \times \boldsymbol{B}]_{i}=} & -\mu_{0} \frac{\partial P}{\partial u^{i}}+\frac{B}{\sqrt{g_{33}}} \frac{\partial b_{i}}{\partial u^{3}} \\
& +\frac{\mu_{0} P \sqrt{g_{33}}}{B} \frac{\partial}{\partial u^{i}}\left(\frac{B}{\sqrt{g_{33}}}\right) .
\end{aligned}
$$

Using Eqs. (3) and (28)-(30), the first equation in Eq. (34), and the relation $\varpi=R+O(\varepsilon)$ we obtain

$[(\nabla \times \boldsymbol{B}) \times \boldsymbol{b}]_{i}=\frac{2 \varepsilon^{2} q R^{2} B \sigma}{q^{2}+R^{2}} \epsilon_{i 3 j} b^{j}[1+O(\varepsilon)]$.

Substituting Eqs. (47) and (48) in Eq. (39), taking into account that, in accordance with Eqs. (28), (29) and (31), the ratio of the last term on the right-hand side of Eq. (47) to the first term is of the order of $\varepsilon$, and using Eq. (38) yields

$$
\begin{aligned}
\rho \frac{\partial^{2} \bar{\xi}_{1}}{\partial t^{2}}= & -\varepsilon^{-1} \frac{\partial P}{\partial \sigma}+\frac{\varepsilon^{-1} B}{\mu_{0} \sqrt{q^{2}+R^{2}}} \frac{\partial b_{1}}{\partial \varphi} \\
& -\frac{2 \varepsilon q R^{2} B \sigma}{\mu_{0}\left(q^{2}+R^{2}\right)} b^{2}+O(\varepsilon), \\
\rho \frac{\partial^{2} \bar{\xi}_{2}}{\partial t^{2}}= & -\varepsilon^{-1} \frac{\partial P}{\partial \theta}+\frac{\varepsilon^{-1} B}{\mu_{0} \sqrt{q^{2}+R^{2}}} \frac{\partial b_{2}}{\partial \varphi} \\
& +\frac{2 \varepsilon q R^{2} B \sigma}{\mu_{0}\left(q^{2}+R^{2}\right)} b^{1}+O(\varepsilon) .
\end{aligned}
$$

It follows from Eqs. (49) and (50) that $P=O(\varepsilon)$. Then it follows from Eqs. (28) and (45) that

$$
\frac{1}{\sqrt{g}}\left[\frac{\partial}{\partial u^{1}}\left(B \bar{\xi}^{1} \sqrt{\frac{g}{g_{33}}}\right)+\frac{\partial}{\partial u^{2}}\left(B \bar{\xi}^{2} \sqrt{\frac{g}{g_{33}}}\right)\right]=O\left(\varepsilon^{2}\right) .
$$

Using Eqs. (28), (29), (31) and (51), the estimate $\xi^{3}=O(\varepsilon)$, and the relation $b_{i}=g_{i j} b^{j}$ we obtain from Eq. (42)

$b^{i}=\frac{\varepsilon^{-1} B}{\sqrt{q^{2}+R^{2}}} \frac{\partial \bar{\xi}^{i}}{\partial \varphi}+O(1), \quad(i=1,2)$,

$$
b_{1}=\frac{\varepsilon B R^{2}}{\sqrt{q^{2}+R^{2}}} \frac{\partial \bar{\xi}^{1}}{\partial \varphi}+O\left(\varepsilon^{2}\right), \quad b_{2}=\frac{\varepsilon B R^{2} \sigma^{2}}{\sqrt{q^{2}+R^{2}}} \frac{\partial \bar{\xi}^{2}}{\partial \varphi}+O\left(\varepsilon^{2}\right) .
$$

Substituting Eqs. (52) and (53) in Eqs. (49) and (50) we transform them to

$$
\begin{aligned}
\rho \frac{\partial^{2} \bar{\xi}_{1}}{\partial t^{2}}=-\varepsilon^{-1} \frac{\partial P}{\partial \sigma} & +\frac{B^{2} R^{2}}{\mu_{0}\left(q^{2}+R^{2}\right)} \frac{\partial^{2} \bar{\xi}^{1}}{\partial \varphi^{2}} \\
& -\frac{2 q R^{2} B^{2} \sigma}{\mu_{0}\left(q^{2}+R^{2}\right)^{3 / 2}} \frac{\partial \bar{\xi}^{2}}{\partial \varphi}+O(\varepsilon),
\end{aligned}
$$

$$
\begin{aligned}
\rho \frac{\partial^{2} \bar{\xi}_{2}}{\partial t^{2}}=-\varepsilon^{-1} \frac{\partial P}{\partial \theta} & +\frac{B^{2} R^{2} \sigma^{2}}{\mu_{0}\left(q^{2}+R^{2}\right)} \frac{\partial^{2} \bar{\xi}^{2}}{\partial \varphi^{2}} \\
& +\frac{2 q R^{2} B^{2} \sigma}{\mu_{0}\left(q^{2}+R^{2}\right)^{3 / 2}} \frac{\partial \bar{\xi}^{1}}{\partial \varphi}+O(\varepsilon) .
\end{aligned}
$$

Let us introduce the length along the loop axis,

$s=\sqrt{q^{2}+R^{2}}\left(\varphi-\varphi_{0}\right)$.

Using Eqs. (28), (35), (36) and (38) we obtain the relations

$\bar{\xi}^{1}=\frac{\xi_{r}}{R}+O(\varepsilon), \quad \bar{\xi}^{2}=\frac{\xi_{\theta}}{R \sigma}+O(\varepsilon)$,

$\bar{\xi}_{1}=R \xi_{r}+O(\varepsilon), \quad \bar{\xi}_{2}=R \sigma \xi_{\theta}+O(\varepsilon)$.

Substituting Eqs. (57) in (51), (54)and (55), using Eqs. (28) and (56), and returning to the original variable $r$, we obtain in the leading order approximations with respect to $\varepsilon$ the following system of equations,

$\frac{\partial\left(r \xi_{r}\right)}{\partial r}+\frac{\partial \xi_{\theta}}{\partial \theta}=0$

$\frac{\partial^{2} \xi_{r}}{\partial t^{2}}-V_{A}^{2} \frac{\partial^{2} \xi_{r}}{\partial s^{2}}=-\frac{1}{\rho} \frac{\partial P}{\partial r}-\frac{2 q V_{A}^{2}}{q^{2}+R^{2}} \frac{\partial \xi_{\theta}}{\partial s}$,

$\frac{\partial^{2} \xi_{\theta}}{\partial t^{2}}-V_{A}^{2} \frac{\partial^{2} \xi_{\theta}}{\partial s^{2}}=-\frac{1}{r \rho} \frac{\partial P}{\partial \theta}+\frac{2 q V_{A}^{2}}{q^{2}+R^{2}} \frac{\partial \xi_{r}}{\partial s}$

Note that the plasma density $\rho$ and $V_{A}$ in general depend on $s$. This system of equations has to be supplemented with the boundary conditions. It is straightforward to show that the normal vector to the tube boundary is given by $\boldsymbol{n}=\boldsymbol{e}_{r}+O(\varepsilon)$. Hence, in the leading order approximations with respect to $\varepsilon$, we can take $\boldsymbol{n}=\boldsymbol{e}_{r}$. The normal component of the displacement is approximately equal to $\xi_{r}$. Recall that the equation of the tube boundary in the curvilinear coordinates is $r=a$. Let us introduce the jump of a function $f$ across the boundary,

$\llbracket f \rrbracket=\lim _{\delta \rightarrow+0}\{f(a+\delta)-f(a-\delta)\}$.

The perturbation of the total pressure and the normal component of the displacement have to be continuous at the boundary. These conditions are written as

$\llbracket P \rrbracket=0, \quad \llbracket \xi_{r} \rrbracket=0 \quad$ at $\quad r=a$.

Note that, since the equilibrium magnetic field is inhomogeneous, we have to impose the condition that the Lagrangian perturbation of the total pressure is continuous at the boundary. 
However, the equilibrium magnetic field and its partial derivatives with respect to $r, \theta$ and $s$ are continuous at the boundary. This implies that the jump in the Lagrangian perturbation of the total pressure coincides with the jump in the Eulerian perturbation of the total pressure. Finally, since the magnetic field lines are frozen in the dense photospheric plasma at the loop ends, we have

$\xi_{r}=\xi_{\theta}=0 \quad$ at $\quad s=0, L$,

where

$L=\sqrt{q^{2}+R^{2}}\left(\pi-2 \varphi_{0}\right)$

is the length of the loop. The system of Eqs. (58)-(60) together with the boundary conditions Eqs. (61) and (62) will be used in the next section to derive the governing equation for the loop kink oscillations.

\section{Derivation of governing equation}

The aim of this section is to derive the governing equation for kink oscillations. It follows from Eq. (58) that $\xi_{r}$ and $\xi_{\theta}$ can be written in terms of one function,

$\xi_{r}=\frac{1}{r} \frac{\partial \psi}{\partial \theta}, \quad \xi_{\theta}=-\frac{\partial \psi}{\partial r}$.

Substituting these expressions in Eqs. (59) and (60) and eliminating $P$ from the obtained equations using cross-differentiation we obtain the equation for $\psi$,

$\frac{\partial^{2} F}{\partial t^{2}}-V_{A}^{2} \frac{\partial^{2} F}{\partial s^{2}}=0, \quad F=r \frac{\partial}{\partial r} r \frac{\partial \psi}{\partial r}+\frac{\partial^{2} \psi}{\partial \theta^{2}}$.

It follows from Eqs. (62) and (64) that

$\psi=0 \quad$ at $\quad s=0, L$

In what follows we only consider eigenmodes of kink oscillations and take the displacement proportional to $\exp (-i \omega t)$. Then the first equation in Eq. (65) reduces to

$\frac{\partial^{2} F}{\partial s^{2}}+\frac{\omega^{2}}{V_{A}^{2}} F=0$

This equation describes Alfvén oscillations of individual magnetic field lines. We assume that the eigenfrequencies of kink oscillations do not coincide with any local Alfvén frequency. Then Eq. (67) has only a trivial solution, $F=0$. Therefore $\psi$ satisfies the equation

$r \frac{\partial}{\partial r} r \frac{\partial \psi}{\partial r}+\frac{\partial^{2} \psi}{\partial \theta^{2}}=0$

We restrict our analysis to kink oscillations and take $\psi$ proportional to $\exp (i \theta)$, so that this equation reduces to

$r \frac{\partial}{\partial r} r \frac{\partial \psi}{\partial r}-\psi=0$

Substituting Eq. (64) in Eq. (60) we obtain

$P=\rho\left(i r V_{A}^{2} \frac{\partial^{3} \psi}{\partial r \partial s^{2}}+i r \omega^{2} \frac{\partial \psi}{\partial r}+\frac{2 q V_{A}^{2}}{q^{2}+R^{2}} \frac{\partial \psi}{\partial s}\right)$.
Using Eqs. (64) and (70) we rewrite the boundary conditions Eq. (61) at $r=a$ in terms of $\psi$,

$\llbracket \psi \rrbracket=0 \quad$ at $\quad r=a$,

$\llbracket \rho\left(V_{A}^{2} \frac{\partial^{3} \psi}{\partial r \partial s^{2}}+\omega^{2} \frac{\partial \psi}{\partial r}-\frac{2 i q V_{A}^{2}}{a\left(q^{2}+R^{2}\right)} \frac{\partial \psi}{\partial s}\right) \|=0$ at $r=a$.

The solution to Eq. (69) regular at $r=0$, vanishing as $r \rightarrow \infty$, and satisfying Eq. (71) is straightforward,

$\psi=\Psi(s) \begin{cases}r, & r<a, \\ a^{2} / r, & r>a,\end{cases}$

where, at present, $\Psi(s)$ is an arbitrary function satisfying $\Psi(s)=$ 0 at $s=0, L$. Substituting Eq. (73) in Eq. (72) and taking into account that $\llbracket \rho V_{A}^{2} \rrbracket=\llbracket B^{2} / \mu_{0} \rrbracket=0$ we obtain the governing equation for $\Psi(s)$,

$\frac{\mathrm{d}^{2} \Psi}{\mathrm{d} s^{2}}+\frac{\omega^{2}}{C_{k}^{2}} \Psi=0, \quad C_{k}^{2}=\frac{2 B^{2}}{\mu_{0}\left(\rho_{e}+\rho_{i}\right)}$.

Since the plasma density varies along the loop, $C_{k}$ is a function of $s$. The function $\Psi$ satisfies the boundary conditions

$\Psi=0 \quad$ at $\quad s=0, L$.

Equations (74) and (75) constitute the boundary-value problem that determines the frequencies of the fundamental mode and overtones of kink oscillations of the curved non-planar loop. This boundary-value problem coincides exactly with the boundary-value problem determining the frequencies of kink oscillations of a straight magnetic loop with the density varying along the loop (see Dymova \& Ruderman 2005). Hence, the loop curvature and non-planarity does not affect directly the frequencies of kink oscillations. This result can be expected on the physical ground because, in the leading order approximation with respect to $\varepsilon$, we neglect both the curvature and torsion of the tube. This effectively reduces the problem to studying kink oscillations of a straight tube. However, the loop curvature and nonplanarity can affect the oscillation frequencies indirectly because they determine the dependence of $\rho_{e}$ and $\rho_{i}$ on $s$.

It follows from Eqs. (64) and (73) that $\xi_{r}=i \Psi$ and $\xi_{\theta}=-\Psi$ inside the tube. These relations imply that both $\xi_{r}$ and $\xi_{\theta}$ are independent of $r$ inside the tube. Then $\xi$ is also independent of $r$ inside the tube, so that, in the leading order approximation with respect to $\varepsilon$, the tube oscillates as a solid.

Equation (74) describes only the eigenmode dependence on $s$. We managed to factor out the radial dependence because $\rho_{i}$ and $\rho_{e}$ are independent of $r$. As a result, in the leading order approximation with respect to $\varepsilon$, the Alfvén speed inside the loop and outside the loop in the loop vicinity is independent of $r$. However the magnetic field magnitude is not constant. In accordance with Eq. (2) it is equal to $q B_{0}\left(q^{2}+\varpi^{2}\right)^{-1 / 2}$. Hence it varies from $B_{0}$ at $\varpi=0$ to 0 as $\varpi \rightarrow \infty$. This implies that, in our model, there is the fundamental Alfvén continuum $\left[0, \omega_{A f}\right]$ and the Alfvén continuum for every overtone, $\left[0, \omega_{A n}\right]$, where $n=1,2, \ldots$ We do not give the expressions for $\omega_{A f}$ and $\omega_{A n}$ because they are not used in what follows. It can be shown that the frequency of the fundamental kink mode is less than $\omega_{A f}$, and the frequency of the $n$th overtone is less than $\omega_{A n}$, which implies that there is the Alfvén resonance. This should cause the resonant damping of the loop kink oscillations. However the resonant surface is at the distance of the order of $R \gg a$ from the 
loop, where the oscillation amplitude is extremely small. As a result the resonant damping is very weak and can be neglected.

Since the Alfvén frequency is tending to zero as $\varpi \rightarrow \infty$, at some distance from the loop the eigenmode is leaky, which also should cause the oscillation damping. However, once again, the wave leakage occurs at distances of the order of $R \gg a$ from the loop, where the oscillation amplitude is extremely small. As a result the damping due to leakage is also very weak and can be neglected.

\section{Polarization of kink oscillations}

As we have already mentioned the non-planarity practically does not affect the frequencies of kink oscillations. The main effect of non-planarity resides in changing the polarization of kink oscillations. In this section we discuss possible observational signatures of this change of polarization. The following analysis is a generalization of the analysis of the kink mode polarization in the case of straight magnetic tube given by Ruderman \& Erdélyi (2009) (see Sect. 3 of that paper).

It is impossible to obtain any information about the polarization of kink eigenmodes directly from Eq. (74) because, when deriving Eq. (74), we canceled out the dependence on $t$ and $\theta$ taking all variables proportional to $\exp (-i \omega t+i \theta)$. We need to restore this dependence. To do this we write $\psi=r \Psi(s) \exp (-i \omega t+$ $i \theta$ ) inside the loop. It is obvious that we obtain the same Eq. (74) if we take $\psi=r \Psi(s) \exp ( \pm i \omega t \pm i \theta)$ with any combinations of the signs. Hence the general solution is the linear combination of four different exponents. However the coefficients at these exponents are not arbitrary: they have to satisfy the condition that $\psi$ is a real function. It is easy to show that, under this restriction, the most general form of $\psi$ is

$$
\psi=r \Psi(s) \mathfrak{R}\left\{\exp (i \theta)\left[A_{1} \exp (-i \omega t)+A_{2} \exp (i \omega t)\right]\right\}
$$

where $\mathfrak{R}$ indicates the real part of a quantity, and $A_{1}$ and $A_{2}$ are arbitrary complex constants. Since it follows from Eqs. (74) and (75) that the ratio of the imaginary and real part of $\Psi$ is constant, we can take $\Psi(s)$ to be real without the loss of generality. Substituting Eq. (76) in Eq. (64) we obtain after some algebra

$$
\begin{aligned}
& \xi_{r}=\Psi(s)\left[A_{-} \cos \left(\omega t-\theta+\alpha_{-}\right)+A_{+} \cos \left(\omega t+\theta+\alpha_{+}\right)\right], \\
& \xi_{\theta}=\Psi(s)\left[A_{-} \sin \left(\omega t-\theta+\alpha_{-}\right)-A_{+} \sin \left(\omega t+\theta+\alpha_{+}\right)\right],
\end{aligned}
$$

where $A_{ \pm}$and $\alpha_{ \pm}$are arbitrary real constants.

Let us now calculate the components of $\boldsymbol{\xi}$ inside the tube in Cartesian coordinates. Using Eqs. (25) and (26) we obtain that, in the leading-order approximation with respect to $\varepsilon$, the Cartesian components of the unit vectors in the $r$ - and $\theta$-direction are given by

$$
\begin{aligned}
\boldsymbol{e}_{r}=\frac{\partial \boldsymbol{X}}{\partial \sigma}\left|\frac{\partial \boldsymbol{X}}{\partial \sigma}\right|^{-1}= & \left(\frac{R \cos \theta}{\sqrt{R^{2}+q^{2}}}, \sin \theta \cos \varphi+\frac{q \cos \theta \sin \varphi}{\sqrt{R^{2}+q^{2}}},\right. \\
& \left.\sin \theta \sin \varphi-\frac{q \cos \theta \cos \varphi}{\sqrt{R^{2}+q^{2}}}\right)
\end{aligned}
$$

$$
\begin{aligned}
\boldsymbol{e}_{\theta}=\frac{\partial \boldsymbol{X}}{\partial \theta}\left|\frac{\partial \boldsymbol{X}}{\partial \theta}\right|^{-1}= & \left(-\frac{R \sin \theta}{\sqrt{R^{2}+q^{2}}}, \cos \theta \cos \varphi-\frac{q \sin \theta \sin \varphi}{\sqrt{R^{2}+q^{2}}}\right. \\
& \left.\cos \theta \sin \varphi+\frac{q \sin \theta \cos \varphi}{\sqrt{R^{2}+q^{2}}}\right)
\end{aligned}
$$

Now, using the relation $\boldsymbol{\xi}=\xi_{r} \boldsymbol{e}_{r}+\xi_{\theta} \boldsymbol{e}_{\theta}$ and Eqs. (56) and (77)-(79), we obtain

$$
\begin{aligned}
\xi_{x}=\Psi(s) & \frac{R A_{c} \cos \left(\omega t+\alpha_{c}\right)}{\sqrt{R^{2}+q^{2}}}, \\
\xi_{y}=\Psi(s) & \left\{A_{s} \sin \left(\omega t+\alpha_{s}\right) \cos \left(\varphi_{0}+\frac{s}{\sqrt{R^{2}+q^{2}}}\right)\right. \\
+ & \left.\frac{q A_{c} \cos \left(\omega t+\alpha_{c}\right)}{\sqrt{R^{2}+q^{2}}} \sin \left(\varphi_{0}+\frac{s}{\sqrt{R^{2}+q^{2}}}\right)\right\},
\end{aligned}
$$

$$
\begin{aligned}
\xi_{z}=\Psi(s) & \left\{A_{s} \sin \left(\omega t+\alpha_{s}\right) \sin \left(\varphi_{0}+\frac{s}{\sqrt{R^{2}+q^{2}}}\right)\right. \\
& \left.-\frac{q A_{c} \cos \left(\omega t+\alpha_{c}\right)}{\sqrt{R^{2}+q^{2}}} \cos \left(\varphi_{0}+\frac{s}{\sqrt{R^{2}+q^{2}}}\right)\right\},
\end{aligned}
$$

where $A_{c}, A_{s}, \alpha_{c}$ and $\alpha_{s}$ are expressed in terms of $A_{ \pm}$and $\alpha_{ \pm}$. Since $A_{ \pm}$and $\alpha_{ \pm}$are arbitrary real constants, $A_{c}, A_{s}, \alpha_{c}$ and $\alpha_{s}$ are also arbitrary real constants.

Let us introduce the Frenet basis for the axis of the loop. It consists of the unit tangent vector $\hat{\tau}$, the unit vector of principal normal $\hat{\boldsymbol{n}}$, and the unit binormal vector $\hat{\boldsymbol{b}}$. If $\boldsymbol{X}=\boldsymbol{X}_{a}(s)$ is the equation of the tube axis, then

$\hat{\boldsymbol{\tau}}=\frac{\mathrm{d} \boldsymbol{X}_{a}}{\mathrm{~d} s}, \quad \hat{\boldsymbol{n}}=\frac{\mathrm{d} \hat{\tau}}{\mathrm{d} s}\left|\frac{\mathrm{d} \hat{\tau}}{\mathrm{d} s}\right|^{-1}, \quad \hat{\boldsymbol{b}}=\hat{\boldsymbol{\tau}} \times \hat{\boldsymbol{n}}$.

Using Eqs. (6) and (56) we obtain that the Cartesian coordinates of the Frenet basis vectors are

$$
\begin{array}{r}
\hat{\tau}=\frac{1}{\sqrt{R^{2}+q^{2}}}\left(q,-R \sin \left(\varphi_{0}+\frac{s}{\sqrt{R^{2}+q^{2}}}\right),\right. \\
\left.R \cos \left(\varphi_{0}+\frac{s}{\sqrt{R^{2}+q^{2}}}\right)\right),
\end{array}
$$

$\hat{\boldsymbol{n}}=-\left(0, \cos \left(\varphi_{0}+\frac{s}{\sqrt{R^{2}+q^{2}}}\right), \sin \left(\varphi_{0}+\frac{s}{\sqrt{R^{2}+q^{2}}}\right)\right)$,

$$
\begin{aligned}
\hat{\boldsymbol{b}}=\frac{1}{\sqrt{R^{2}+q^{2}}}(R, q \sin & \left(\varphi_{0}+\frac{s}{\sqrt{R^{2}+q^{2}}}\right), \\
& \left.-q \cos \left(\varphi_{0}+\frac{s}{\sqrt{R^{2}+q^{2}}}\right)\right) .
\end{aligned}
$$

Using Eqs. (80)-(82) and (84)-(86) we obtain that the projection of $\boldsymbol{\xi}$ on the Frenet basis vectors are given by

$\xi_{\tau}=\hat{\boldsymbol{\tau}} \cdot \boldsymbol{\xi}=0, \quad \xi_{n}=\hat{\boldsymbol{n}} \cdot \boldsymbol{\xi}=A_{s} \Psi(s) \sin \left(\omega t+\alpha_{s}\right)$,

$\xi_{b}=\hat{\boldsymbol{b}} \cdot \boldsymbol{\xi}=A_{c} \Psi(s) \cos \left(\omega t+\alpha_{c}\right)$.

Eliminating $t$ from these equations we obtain

$\frac{\left(\xi_{n} \cos \beta-\xi_{b} \sin \beta\right)^{2}}{\left(H_{1} \Psi(s)\right)^{2}}+\frac{\left(\xi_{n} \sin \beta+\xi_{b} \cos \beta\right)^{2}}{\left(H_{2} \Psi(s)\right)^{2}}=1$,

where $\beta$ is defined by

$\tan 2 \beta=\frac{2 A_{s} A_{c} \sin \alpha}{A_{s}^{2}-A_{c}^{2}}, \quad \alpha=\alpha_{c}-\alpha_{s}$, 


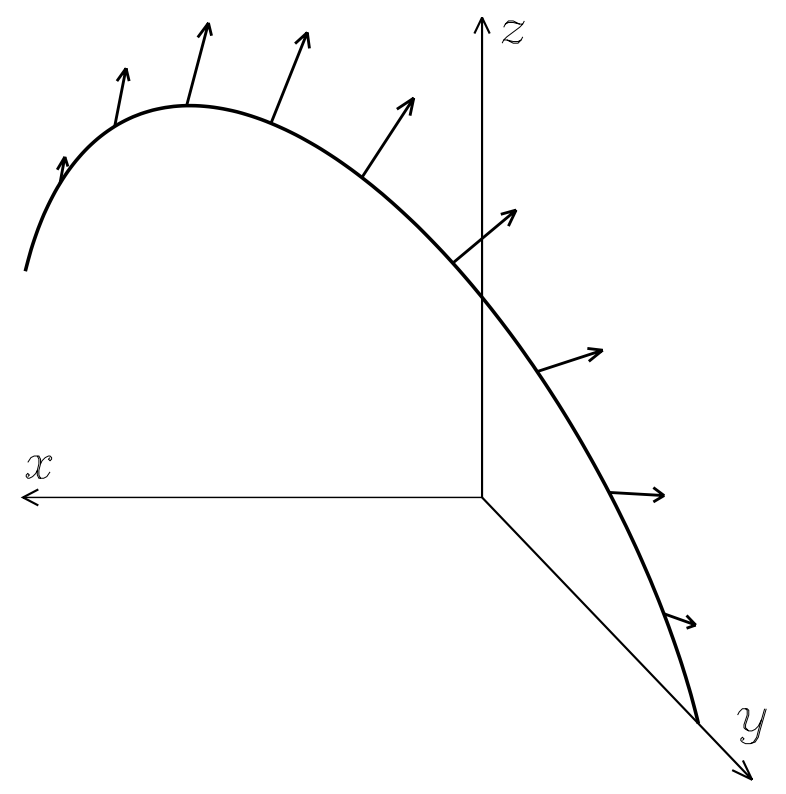

Fig. 4. Illustration of polarization of a fundamental kink mode.

and $H_{1}$ and $H_{2}$ are expressed in terms of $A_{s}, A_{c}, \alpha$ and $\beta$. We do not give these expressions because they are not used in what follows. Equation (88) is the equation of an ellipse in the $\xi_{n} \xi_{b^{-}}$ plane with the half-axes equal to $H_{1} \Psi(s)$ and $H_{2} \Psi(s)$. The angle between one of the axes and vector $\hat{\boldsymbol{n}}$ is $\beta$. Note that this angle is independent of $s$. The ratio of axes is equal to $H_{2} / H_{1}$, so that it is also independent of $s$. We see that, in general, the oscillation is elliptically polarized in the plane spanned by the vectors $\hat{\boldsymbol{n}}$ and $\hat{\boldsymbol{b}}$. This plane is orthogonal to the loop axis. The length of the axes of the polarization ellipse are proportional to $\Psi(s)$. When the plane normal to the loop axis is moving along the loop the axes of the polarization ellipse rotate together with the vectors $\hat{\boldsymbol{n}}$ and $\hat{\boldsymbol{b}}$. As it is well known from differential geometry the angle of rotation per unit length is equal to the loop axis torsion. Using Eq. (6) we easily find that this torsion is equal to $q\left(R^{2}+q^{2}\right)^{-1}$.

The oscillation is linearly polarized when one of the two axes of the polarization ellipse is zero. However, since we do not give the expressions for $H_{1}$ and $H_{2}$, we will use another condition. Obviously the oscillation is linear polarized if $\xi_{n} / \xi_{b}$ is independent of $t$. This condition reduces to $\cos \left(\alpha_{c}-\alpha_{s}\right)=0$, so that $\alpha_{c}=\alpha_{s}+\pi / 2+n \pi$, where $n$ is any integer number. Without loss of generality we can take $\alpha_{c}=\alpha_{s}+\pi / 2$. Then we obtain

$\left(\xi_{n}, \xi_{b}\right)=A(\cos \beta, \sin \beta) \Psi(s) \sin \left(\omega t+\alpha_{s}\right)$,

where

$A=\sqrt{A_{s}^{2}+A_{c}^{2}}, \quad \tan \beta=\frac{A_{c}}{A_{s}}$.

Figure 4 illustrates the polarization of a fundamental kink eigenmode. The small arrows show the loop axis displacement. If the eigenmode is linearly polarized then the direction of the displacement vector at each point on the loop axis remains the same at any moment of time. Hence, the end of the displacement vector is moving along a straight line. In an elliptically polarized eigenmode the direction of polarization vector rotates with constant angular speed about the tangent to the loop axis. The end of the displacement vector is moving along the polarization ellipse.
For a linearly polarized eigenmode we can fix arbitrarily the polarization direction at one point. After that the polarization direction at all other points on the loop axis will be defined. Similarly for an elliptically polarized mode we can fix the direction of the larger half-axis of the polarization ellipse. After that the directions of axes of the polarization ellipse at all other points on the loop axis will be defined. The fact that we can choose the polarization direction at one point arbitrarily implies that kink oscillations of the loop are degenerate. There are infinitely many eigenmodes with different polarization corresponding to the same eigenfrequency. The situation is similar to that in the case of a straight tube: due to the symmetry the tube can oscillate with the same frequency in any direction.

Van Doorsselaere et al. (2004) studied kink oscillations of a loop having a half-circle shape. They found that the account of the loop curvature removes the degeneration of kink oscillations that takes place in the case of a straight tube. Now there are two fundamental eigenmodes, one polarized in the vertical and one in the horizontal direction. These modes have different frequencies. The same is true for all overtones. These results were confirmed by the numerical study by Terradas et al. (2006).

However, Van Doorsselaere et al. (2004) found that the difference in frequencies of the vertically and horizontally polarized eigenmodes is of the order of $\varepsilon^{2}$, so that it is extremely small for any realistic coronal loop. If the loop is initially displaced in the direction that is neither vertical not horizontal then both the vertically and horizontally polarized eigenmodes will be excited. Since they have different frequencies, the beating phenomenon will take place. However, this phenomenon will be manifested only after the time of the order of the oscillation period times $\varepsilon^{-2}$, which is much larger than the characteristic damping time of kink oscillations. This implies that the splitting of oscillation frequency caused by the curvature is unimportant from the observational point of view.

We anticipate that the situation with non-planar loops is similar to that with curved planar loops. Namely, we anticipate that, extending our analysis to the higher order approximation with respect to $\varepsilon$, we obtain that there are two fundamental modes with mutually orthogonal polarization directions and different frequencies. Further, we anticipate that the same is true for all overtones. However the frequency differences will be of the order of $\varepsilon^{2}$ (or, at least, of the order of $\varepsilon$ ), so these differences are unimportant for applications.

\section{Implication on coronal seismology}

The analysis in this section has been inspired by the observation of a kink oscillation of a non-planar loop reported by Schrijver et al. (2002) and interpreted by De Moortel \& Brady (2007). De Moortel \& Brady (2007) found that the observed oscillation contained two harmonics, one with the period 577-672 s, and the other with the period 250-346 s. The amplitude of the harmonic with the larger period was much larger than the amplitude of the harmonic with the smaller period. The most important result obtained by De Moortel \& Brady (2007) was that the dominant harmonic has a node at some point on the loop. This result inspired De Moortel \& Brady (2007) to interpret this harmonic as the first overtone of the loop kink oscillation. They then discussed the possible reasons why the solar flare near the loop excited mainly the first overtone, and not the fundamental harmonic as in the absolute majority of observations of coronal loop kink oscillations. Note that De Moortel \& Brady (2007) did not exclude the possibility that the dominant harmonic is still the 
M. S. Ruderman and A. Scott: Transverse oscillations of non-planar loops

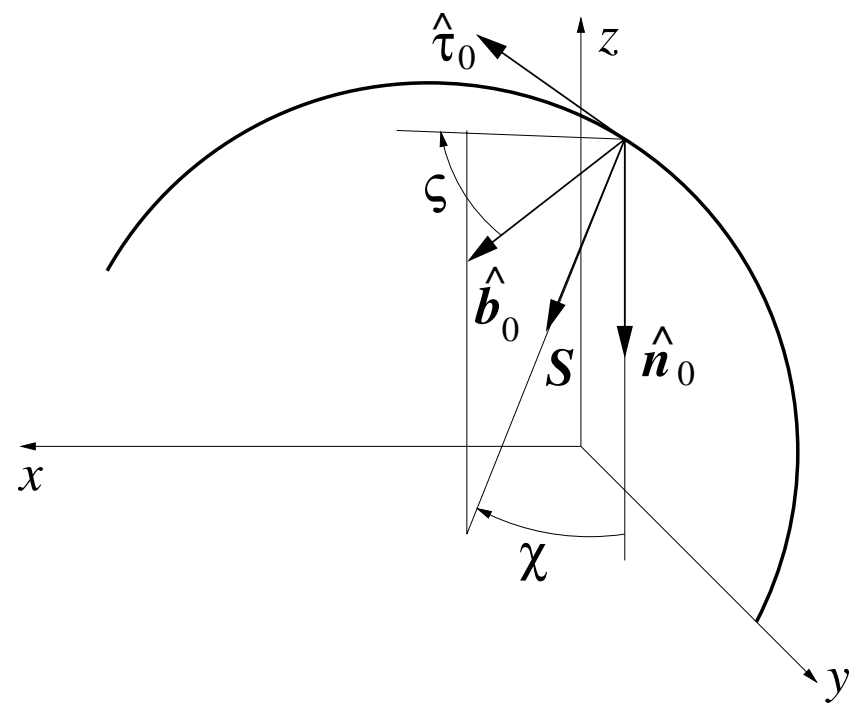

Fig. 5. Introducing the angles $\chi$ and $\varsigma$ is illustrated. The thick line shows the loop axis.

fundamental mode, while the presence of the node is an observational effect related to the loop non-planarity.

In what follows we are not going to apply the results of our theoretical study to the particular kink oscillation of a coronal loop reported by Schrijver et al. (2002) and interpreted by De Moortel \& Brady (2007). Instead we aim to develop a theoretical tool that can be used to answer the question: is the observed kink oscillation of a non-planar loop that has a node the first overtone or, is it the fundamental mode and the presence of the node is an observational effect?

Consider a linearly polarized kink oscillation of a non-planar loop studied in the previous section. Assume that we observe this oscillation and the line-of-sight is determined by the unit vector $\boldsymbol{S}$. Let $\hat{\boldsymbol{\tau}}_{0}, \hat{\boldsymbol{n}}_{0}$ and $\hat{\boldsymbol{b}}_{0}$ be the vectors of the Frenet basis at the loop apex, i.e. at $s=L / 2$. We define vector $S$ as (see Fig. 5)

$\boldsymbol{S}=\hat{\boldsymbol{n}}_{0} \cos \chi+\hat{\boldsymbol{b}}_{0} \sin \chi \cos \varsigma+\hat{\boldsymbol{\tau}}_{0} \sin \chi \sin \varsigma$,

where $\chi \in[0, \pi]$ and $s \in[0,2 \pi]$. Since $s=L / 2$, it follows from Eq. (63) that $\varphi_{0}+s / \sqrt{R^{2}+q^{2}}=\pi / 2$. Substituting this expression in Eqs. (84)-(86) we obtain

$\hat{\boldsymbol{\tau}}_{0}=\frac{(q,-R, 0)}{\sqrt{R^{2}+q^{2}}}, \quad \hat{\boldsymbol{n}}_{0}=(0,0,-1), \quad \hat{\boldsymbol{b}}_{0}=\frac{(R, q, 0)}{\sqrt{R^{2}+q^{2}}}$.

Then we easily obtain

$$
\begin{aligned}
& \hat{\boldsymbol{n}}=\sin \varphi \hat{\boldsymbol{n}}_{0}+\frac{\cos \varphi}{\sqrt{R^{2}+q^{2}}}\left(R \hat{\boldsymbol{\tau}}_{0}-q \hat{\boldsymbol{b}}_{0}\right), \\
& \hat{\boldsymbol{b}}=\frac{q R(1-\sin \varphi) \hat{\boldsymbol{\tau}}_{0}+\left(R^{2}+q^{2} \sin \varphi\right) \hat{\boldsymbol{b}}_{0}}{R^{2}+q^{2}}+\frac{q \cos \varphi}{\sqrt{R^{2}+q^{2}}} \hat{\boldsymbol{n}}_{0},
\end{aligned}
$$

where $\varphi$ is expressed in terms of $s$ by Eq. (56). We can visually observe only the component of vector $\xi$ perpendicular to $S$. It is given by $\xi_{\perp}=\boldsymbol{\xi}-\boldsymbol{S}(\boldsymbol{\xi} \cdot \boldsymbol{S})$. The condition that $\boldsymbol{\xi}_{\perp}=0$ is written as $\boldsymbol{\xi} \| \boldsymbol{S}$. Using Eqs. (90) and (94) we obtain from the condition $\boldsymbol{\xi} \| \boldsymbol{S}$ two equations,

$$
\begin{aligned}
& \frac{\left(R^{2}+q^{2} \sin \varphi\right) \tan \beta}{R^{2}+q^{2}}-\frac{q \cos \varphi}{\sqrt{R^{2}+q^{2}}}= \\
& \tan \chi \cos \varsigma\left(\sin \varphi+\frac{q \cos \varphi \tan \beta}{\sqrt{R^{2}+q^{2}}}\right),
\end{aligned}
$$

$$
\begin{aligned}
& \frac{q R(1-\sin \varphi) \tan \beta}{R^{2}+q^{2}}+\frac{R \cos \varphi}{\sqrt{R^{2}+q^{2}}}= \\
& \tan \chi \sin \varsigma\left(\sin \varphi+\frac{q \cos \varphi \tan \beta}{\sqrt{R^{2}+q^{2}}}\right)
\end{aligned}
$$

Eliminating $\tan \beta$ from Eqs. (95) and (96) we obtain the equation for $\varphi$,

$$
\begin{gathered}
R \sin \varphi \tan \chi(q \cos \varsigma-R \sin \varsigma)+R \sqrt{R^{2}+q^{2}} \cos \varphi \\
-q \tan \chi(R \cos \varsigma+q \sin \varsigma)=0 .
\end{gathered}
$$

If this equation does not have a solution satisfying $\varphi \in\left[\varphi_{0}, \pi-\right.$ $\left.\varphi_{0}\right]$, then this means that, for any polarization angle $\beta$, we cannot have a node in the observation of the loop oscillation in its fundamental mode. This would be strong evidence supporting the conclusion that the observed oscillation is the first overtone.

Let us now assume that Eq. (97) has a solution satisfying $\varphi \in\left[\varphi_{0}, \pi-\varphi_{0}\right]$. In principle, it is possible that there is more than one solution of Eq. (97) satisfying this condition, but we do not consider this case and assume that there is exactly one solution. After solving Eq. (97) we use Eq. (56) to find the position of the node on the loop, $s_{\text {th }}$. Let $s_{\text {obs }}$ be the position of the node found from the observation. If $\left|s_{\mathrm{th}}-s_{\mathrm{obs}}\right|$ is small enough, i.e. if it is in the confidence interval, then it is a strong evidence supporting the conclusion that the observed oscillation is the fundamental mode, while the presence of the node is an observational effect. Contrary, if $\left|s_{\mathrm{th}}-s_{\mathrm{obs}}\right|$ is beyond the confidence interval, then it is an evidence in favour of concluding that the observed oscillation is the first overtone.

Let us consider one example. Assume that we have observed kink oscillations of a non-planar loop described by our model with $R=q, \varphi_{0}=0$ (so that the projection of the loop on the $y z-$ plane is a half-circle), and the line-of-sight vector $S$ is defined by $\chi=\pi / 4$ and $\varsigma=\pi / 3$. Then Eq. (97) reduces to

$(1-\sqrt{3}) \sin \varphi+2 \sqrt{2} \cos \varphi-(1+\sqrt{3})=0$.

The solution to this equation satisfying $\varphi \in(0, \pi)$ is

$\varphi=\arcsin \frac{2 \sqrt{4-2 \sqrt{3}}-1}{6-\sqrt{3}} \approx 0.109$,

so that $s_{\text {th }}=\varphi / \pi \approx 0.035 L$. Hence, if $s_{\text {obs }}$ is close to $0.035 L$ then we conclude that the observed oscillation was the fundamental mode. If, on the other hand, $s_{\text {obs }}$ differs very much from $0.035 L$, then we conclude that the observed oscillation was the first overtone.

\section{Summary and conclusions}

In this paper we have studied the kink oscillations of non-planar coronal magnetic loops. We suggested a simple model of a nonplanar loop with the loop axis being a part of a helical line. The loop cross-section is everywhere a circle of radius $a$, where $a$ is much smaller than the loop curvature radius. The density inside the loop is larger than outside the loop, and both densities inside and outside the loop can vary along the loop. However they do not vary in the transversal directions.

We introduced a curvilinear coordinate system where the loop boundary is a coordinate surface and the loop axis is a coordinate line. Then we wrote the ideal linearized MHD equations and boundary conditions in the curvilinear coordinates. We used these equations and boundary conditions to 
derive the governing equation for the loop kink oscillations. To do this we used the asymptotic method similar to one developed by Dymova \& Ruderman (2005) for the derivation of the governing equation for kink oscillations of a straight magnetic tube with the density varying along the tube. It turns out that our equation is exactly the same as one derived by Dymova \& Ruderman (2005). This implies that, similar to the loop curvature, the loop torsion cannot affect the eigenfrequencies of the non-planar loop oscillations directly. It can only affect them indirectly through modifying the dependence of the density on the distance along the loop.

The main effect of the loop torsion is the variation of the oscillation polarization along the loop. The loop displacement is everywhere orthogonal to the loop axis. In general, at each point on the loop axis the displacement is elliptically polarized in the plane orthogonal to the axis. The ratio of the polarization ellipse axes does not vary along the loop, and the ellipse axes constitute the same angle with the principal normal to the loop axis at any point of the loop axis. Hence, they rotate together with the principal normal due to the loop torsion. We obtain a linearly polarized oscillation in a particular case when one of the polarization ellipse axes is zero. In that case the polarization direction everywhere constitutes the same angle with the principal normal.

We discussed the application of the obtained results to coronal seismology. For this we considered the observation of a linearly polarized kink oscillation of a non-planar coronal loop with the line-of-sight defined by the vector $\boldsymbol{S}$. We assumed that the kink oscillation is the fundamental mode, so that there are no nodes except at the foot points. Visually we can observe only the loop displacement orthogonal to $S$. Hence, we see the node at the point where the loop displacement is parallel to $S$. We derived the equation (see Eq. (97)) that defines the position where the loop displacement is parallel to $S$ under the assumption that the loop oscillation is plane polarized. If this equation has a solution corresponding to a point on the loop axis than we always can find the corresponding polarization direction. We suggest comparing the position of this point with the position of the node found from the observations. If the two positions are close to each other, then this is a strong evidence in favour of the conclusion that the loop oscillates in its fundamental mode and the presence of the node is an observational effect. On the other hand, if the two positions are strongly different, then it is most probable that the node presence indicates that the loop oscillates in its first overtone.

Acknowledgements. M.S.R. acknowledges the support by an STFC grant. A.S. acknowledges the support by an STFC postgraduate fellowship.

\section{References}

Aschwanden, M. J., Wülser, J. P., Nitta, N. V., \& Lemen, J. R. 2008, ApJ, 679, 827

De Moortel, I., \& Brady, C. S. 2007, ApJ, 664, 1210

Dymova, M. V., \& Ruderman, M. S. 2005, Sol. Phys., 229, 79

Edwin, P. M., \& Roberts, B. 1983, Sol. Phys., 88, 179

Korn, G., \& Korn, T. 1961, Mathematical handbook for scientists and engineers (New York: McGraw-Hill)

Riley, K. F., Hobson, M. P., \& Bence, S. J. 2002, Mathematical methods for physics and engineering (Cambridge University Press)

Ruderman, M. S., \& Erdélyi, R. 2009, Space. Sci. Rev., 149, 199

Ryutov, D. D., \& Ryutova, M. P. 1976, Sov. Phys. JETP, 43, 491

Schrijver, C. J., Aschwanden, M. J., \& Tilte, A. M. 2002, Sol. Phys., 206, 59

Terradas, J., Oliver, R., \& Ballester, J. L. 2006, ApJ, 650, L91

Van Doorsselaere, T., Debosscher, A., Andries, J., \& Poedts, S. 2004, A\&A, 424, 1065

Van Doorsselaere, T., Verwichte, E., \& Terradas, J. 2009, Space. Sci. Rev., 149, 299 